\title{
Nonlinear Polarizabilities of Donor-Acceptor Substituted Conjugated Polyenes
}

\author{
Guanhua Chen and Shaul Mukamel* \\ Department of Chemistry, University of Rochester, Rochester, New York 14627
}

Received: January 9, 1996; In Final Form: April 11, $1996^{\otimes}$

\begin{abstract}
The polarizabilities of conjugated polyenes with 8-36 carbon atoms are calculated using the time dependent Hartree-Fock (TDHF) technique. By examining the reduced single-electron density matrix, we explore the relations among the ground state chemical bonding structure and off-resonant and resonant susceptibilities. The effects of a donor-acceptor substitution on oligomers of different sizes are investigated.
\end{abstract}

\section{Introduction}

Linear and nonlinear optical polarizabilities provide an important tool in the investigation of the electronic structure of conjugated molecules. $^{1-7}$ The TDHF technique ${ }^{8}$ assumes that the wave function can be approximated by a single Slater determinant and has been used to calculate the wave function perturbatively in the external fields. Thus, the necessary computation effort is greatly reduced. Alternatively, the TDHF equation can be derived by starting with the equation of motion for a reduced single-electron density matrix. This procedure avoids calculating the many-body wave functions. ${ }^{9-12}$ We have shown how the time dependent reduced single-electron density matrix obtained in this procedure can be used to develop a clear physical insight for the mechanism of optical nonlinearities in terms of collective electronic normal modes. In a recent study based on the TDHF and the Pariser-Parr-Pople (PPP) Hamiltonian of the off-resonant susceptibilities of a donor-acceptor substituted hexatriene, ${ }^{13}$ we showed how the ground state chemical structure can be tuned by donor-acceptor substitutions or application of external static electric fields and we demonstrated how these changes affect the off-resonant polarizabilities, in agreement with experimental results. ${ }^{7}$ In this article we use the same method to investigate the susceptibilities of larger polymers (up to 36 carbon atoms) and explore the structureproperty relations for both off-resonant and resonant polarizabilities. A metastable structure predicted here is found to possess very large nonlinear off-resonant polarizabilities.

\section{Ground State Structure and the Reduced Density Matrix}

We use the PPP Hamiltonian, which is known to capture the essential electronic properties of the $\pi$ electronic system. ${ }^{1,11,14-16}$

The model has one $\pi$ orbital on each site, and the nuclear charge on a site is taken to be $+e$ ( $-e$ is the electronic charge). The nuclear charges on the donor and the acceptor located at both ends of the chain are taken to be $+2 e$ and 0 , respectively. ${ }^{12,13}$ The chain has $N$ sites $(N-2$ bridge sites, one donor and one acceptor) and $N$ electrons. This model has been employed to investigate nonlinear optical properties of donoracceptor substituted hexatriene. ${ }^{13}$ The satisfactory comparison with experimental results ${ }^{7}$ demonstrated that this model captures the essence of donor-acceptor substituted polyenes. We denote $\epsilon_{1}=\epsilon_{\mathrm{D}}, \epsilon_{N}=\epsilon_{\mathrm{A}}$ and all other atomic energies are zero, i.e., $\epsilon_{n}$ $=0(n=2, \ldots, N-1)$. In all calculations we used a symmetric substitution $\epsilon_{\mathrm{D}}=-\epsilon_{\mathrm{A}}$. All parameters are identical with those given in refs 12 and 13 . We define the density matrix operator

${ }^{\otimes}$ Abstract published in Advance ACS Abstracts, May 15, 1996.

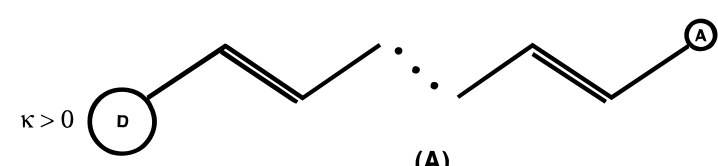

(A)

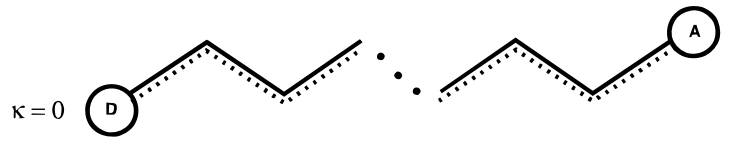

(B)

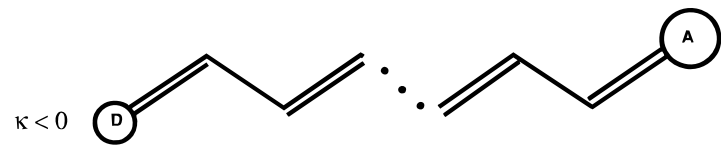

(C)

$$
\begin{array}{llllllllll}
1 & 2 & 3 & 4 & \ldots \ldots & \mathrm{N}-3 & \mathrm{~N}-2 & \mathrm{~N}-1 & \mathrm{~N}
\end{array}
$$

Figure 1. Typical structure of a donor-acceptor substituted polyene: (A) $\kappa<0$; (B) $\kappa=0$; (C) $\kappa<0$.

$\hat{\rho}_{n m}=a_{m}^{+} a_{n} \cdot a_{n}^{+}\left(a_{n}\right)$ is an electron creation (annihilation) operator at the $n$th site. We restrict the analysis to the singlet manifold and eliminate the spin variables. It has been shown that by variation of the donor-acceptor energies, it is possible to control the electronic structure. $7,17,18$ In Figure 1 we show three typical structures of donor-acceptor substituted polyenes. To characterize these structures, we use the bond order alternation (BOA), defined as

$$
\begin{aligned}
\kappa & \equiv 2\left\langle\rho_{n, n+1}-\rho_{n+1, n+2}\right\rangle \\
& \equiv \frac{2}{N-4} \sum_{i=2,4, \ldots, n-4}\left(\rho_{i, i+1}+\rho_{i+2, i+3}-2 \rho_{i+1, i+2}\right)
\end{aligned}
$$

Structures A, B, and $\mathrm{C}$ in Figure 1 are presented with a positive, 0 , and negative $\kappa$, respectively. We have calculated the geometry-optimized Hartree-Fock ground state. By varying $\epsilon_{\mathrm{D}}\left(\right.$ and $\left.\epsilon_{\mathrm{A}}\right)$, we obtained different structures, i.e., different values of BOA. As $\epsilon_{\mathrm{D}}$ increases, the structure evolves from A through $\mathrm{B}$ to $\mathrm{C}$ (Figure 1). In Figure 2 we plot BOA vs $\epsilon_{\mathrm{D}}$ for different $N$. For small $N(10-20)$ BOA changes continuously with $\epsilon_{\mathrm{D}}$. When $N$ is larger $(\geq 22)$, some ranges of BOA cannot be attained and $\kappa$ jumps from 0.4 to -0.4 at $\epsilon_{\mathrm{D}} \approx 3 \mathrm{eV}$. This is consistent with the "forbidden region" observed in ref 19 . The dramatic change of BOA is also the signature of structural change accompanied by a sudden electron transfer from the donor to 


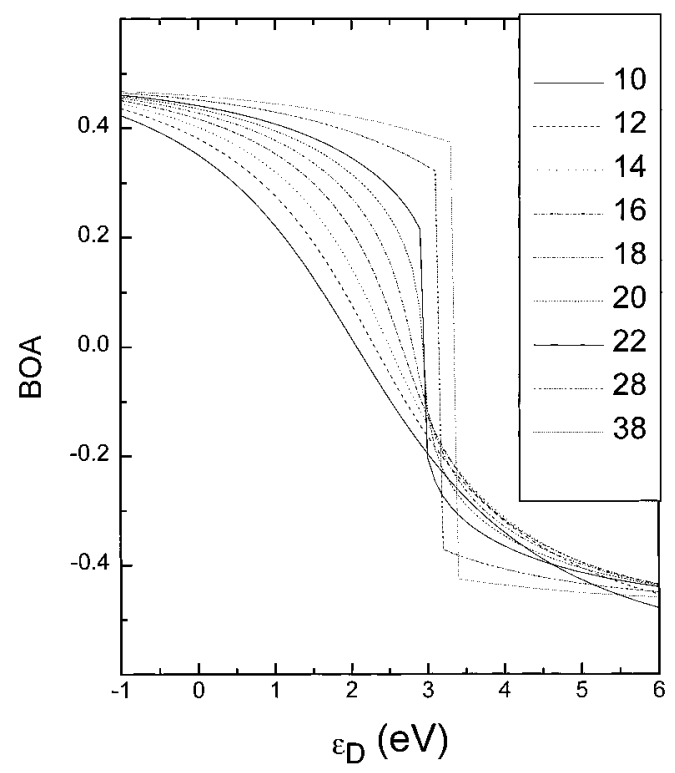

Figure 2. BOA vs $\epsilon_{\mathrm{D}}$ for $N=10 \rightarrow 38$.

TABLE 1: Charges on the Donor $Q_{\mathrm{D}}(\text { in e })^{a}$

\begin{tabular}{cccccccc}
\hline$N=10$ & 14 & 18 & 22 & 26 & 30 & 34 & 38 \\
\hline 0.86 & 0.85 & 0.84 & 0.72 & 0.68 & 0.67 & 0.66 & 0.66 \\
0.91 & 0.90 & 0.91 & 0.95 & 0.99 & 1.00 & 1.01 & 1.01
\end{tabular}
$\mathrm{eV}$.

acceptor. In Table 1 we list the donor charge $Q_{\mathrm{D}}$ at $\epsilon_{\mathrm{D}}=2.9$ and $3.1 \mathrm{eV}$ for systems with different sizes. The charge at the acceptor $Q_{\mathrm{A}}$ is $-Q_{\mathrm{D}}$. The charge transfer is gradual for smaller systems $(N=10 \rightarrow 20)$, and it becomes more abrupt for the larger systems.

Geometry optimization is often complicated by the possibility of reaching a local (as opposed to the global) minimum. To test this, we have in each case performed the optimization twice, starting with two different geometries. We first started with an A-like structure $(\mathrm{BOA} \approx 0.5)$ and then increased the donoracceptor strength (raise $\epsilon_{\mathrm{D}}$ ) incrementally, solving for the Hartree-Fock ground state at each value (I). In the second calculation (II) we started with a C-like structure $(\mathrm{BOA} \approx-0.5)$ and decreased the donor-acceptor strength (lower $\epsilon_{\mathrm{D}}$ ). At each $\epsilon_{\mathrm{D}}$, we used the optimized structure and ground state reduced density matrix corresponding to the previous value of $\epsilon_{\mathrm{D}}$ as the initial guess. For $N=10 \rightarrow 20$, the two calculations resulted in the same optimized structure. However, for $N=22 \rightarrow 38$, over a certain range of donor-acceptor strength that depends on $N$, the two calculations resulted in two different structures. For instance, for $N=30$, the two structures with different BOA appear between $\epsilon_{\mathrm{D}}=2.0$ and $4.2 \mathrm{eV}$. One structure is the ground state and the other is a metastable structure. They are either A-like and C-like or C-like and A-like structures, respectively (see Figure 1). These two states correspond to the Hartree-Fock ground states for both A- and C-like structures and, thus, are two different minima on the ground state potential curve, although their electronic wave functions are quite different (C-like structure is a charge transfer state). To distinguish them, we evaluated the total (electronic and nuclear) energy of the two geometries. In Table 2 we list the energies obtained in the two calculations for various $N$ at $\epsilon_{\mathrm{D}}=3.10 \mathrm{eV}$.

We investigated the energetics as function of geometry for a system $(N=24 \rightarrow 38)$, choosing an $\epsilon_{\mathrm{D}}$ so that the two minima are nearly degenerate. These values are listed in Table 3 . We then constructed new structures along the following path.

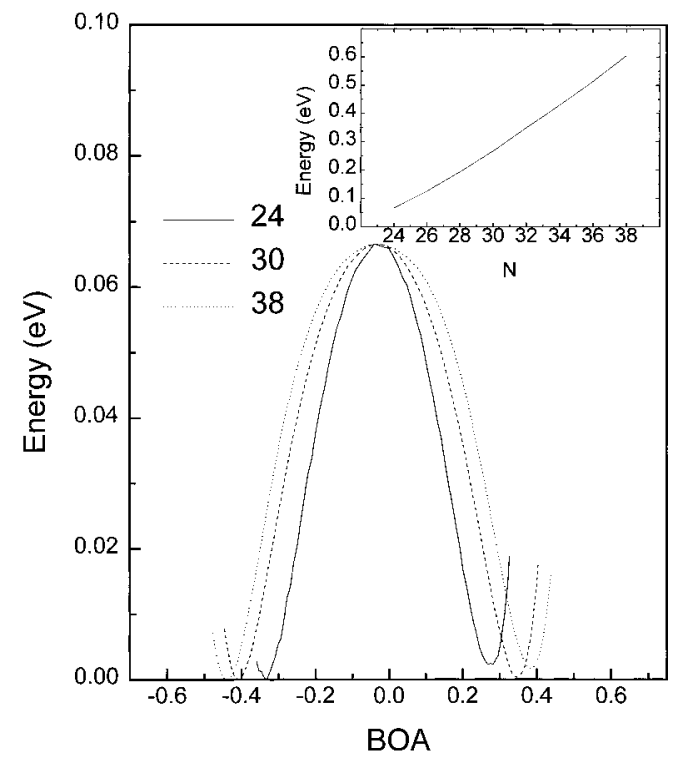

Figure 3. Energy curves for $N=24 \rightarrow 38: N=24$ (solid line); $N=$ 30 (dashed); $N=38$ (dotted). The curves are normalized to the same height. The inset shows the variation of barrier height with $N$.

TABLE 2: Energies of Ground (Top Line) and Metastable (Bottom Line) States (in eV) for $\epsilon_{\mathrm{D}}=3.10 \mathrm{eV}$

\begin{tabular}{ccccc}
\hline$N=22$ & 24 & 26 & 28 & 30 \\
\hline-224.265 & -244.543 & -264.841 & -285.172 & -305.509 \\
-224.223 & -244.473 & -264.833 & -285.136 & -305.447 \\
\hline$N=32$ & 34 & 36 & 38 \\
\hline-325.850 & -346.194 & -366.538 & -386.888 \\
-325.764 & -346.088 & -366.413 & -386.744
\end{tabular}

TABLE 3: Values of $\epsilon_{\mathrm{D}}$ (in eV) Used in Calculating the Energy Barrier between the Ground and Metastable States for Different $N$

\begin{tabular}{lllllllll}
\hline$N$ & 24 & 26 & 28 & 30 & 32 & 34 & 36 & 38 \\
$\epsilon_{\mathrm{D}}$ & 3.05 & 3.10 & 3.15 & 3.20 & 3.25 & 3.28 & 3.30 & 3.35
\end{tabular}

Denoting the bond lengths between the $n$th and $(n+1)$ th site in both geometries $b_{1, n}$ and $b_{2, n}$, respectively, the bond length $b_{n}$ was varied as

$$
b_{n}=b_{1, n}+\lambda\left(b_{2, n}-b_{1, n}\right)
$$

As $\lambda$ is varied between 0 and 1 , the structure changes from $b_{1, n}$ to $b_{2, n}$. At various $\lambda$ we solved for the Hartree-Fock ground state using the same $\epsilon_{\mathrm{D}}$ (with no geometry optimization) and calculated its BOA and energy. In Figure 3 we plot the energies vs BOA for $N=24,30$, and 38. The inset shows the barrier heights for different $N$. The barrier between two minima is $0.06 \mathrm{eV}$, i.e., $700 \mathrm{~K}$ for $N=24$. The metastable geometry is thus accessible at room temperatures.

\section{Off-Resonant Polarizabilities}

Within the dipole approximation the interaction between the $\pi$ electrons and an external electric field $\mathrm{E}(t)$, polarized along the chain $z$ axis, is

$$
H_{\mathrm{ext}}=-\mathrm{E}(t) \mathrm{P}^{\wedge}
$$

where $\mathrm{P}^{\wedge}$ is the dipole moment operator

$$
\hat{\mathrm{P}}=-e \sum_{n, \sigma} z(n) \hat{\rho}_{n n}^{\sigma}
$$

and $z(n)$ is the $z$ coordinate of the $n$th atom. 

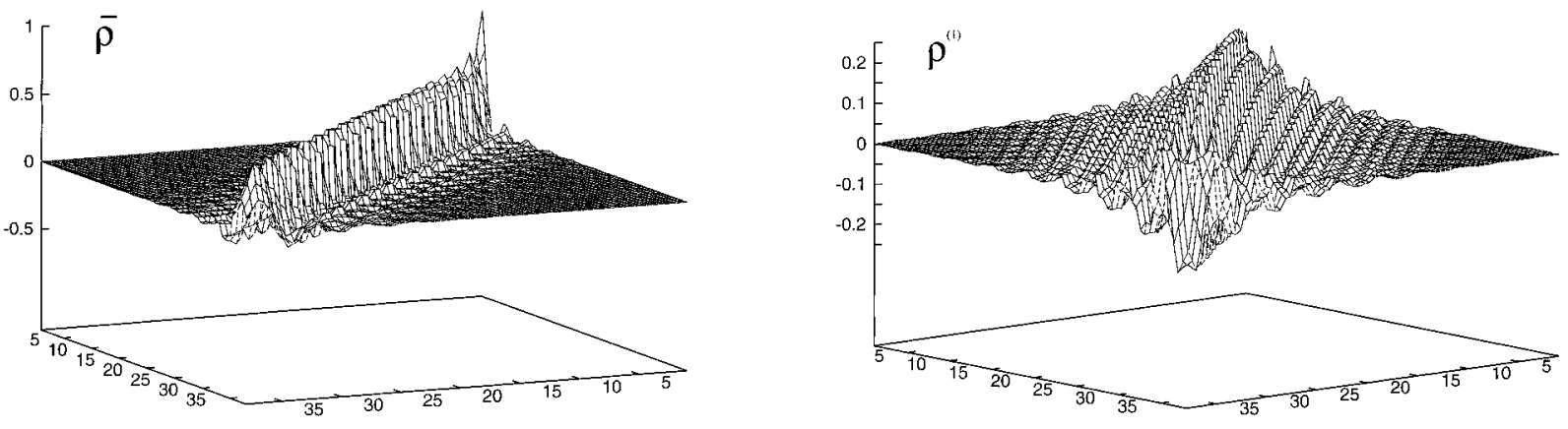

second order
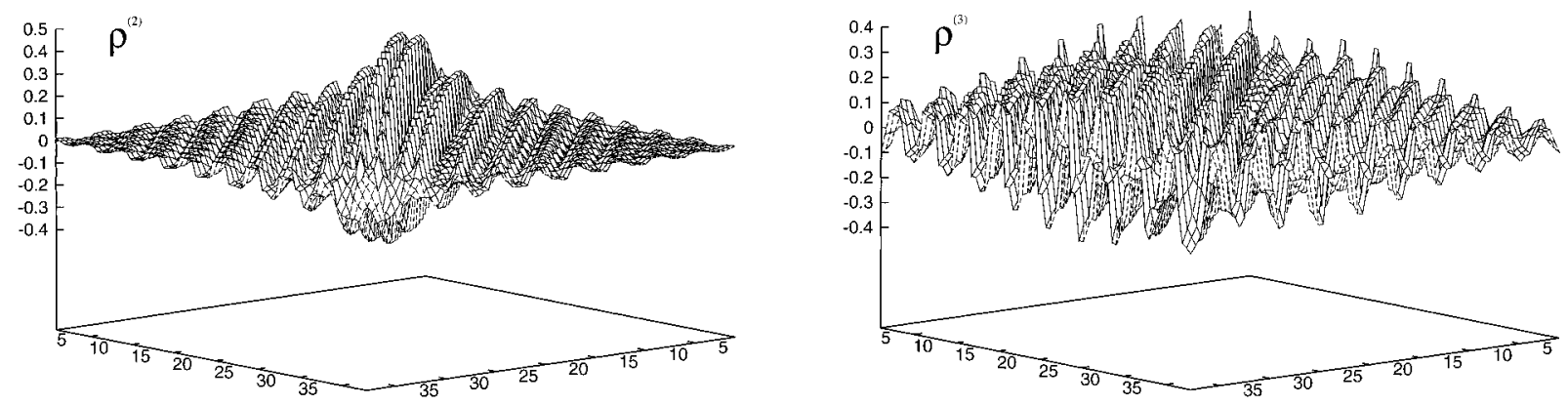

Figure 4. Density matrices $\bar{\rho}, \rho^{(1)}, \rho^{(2)}$, and $\rho^{(3)}$ for $N=38$ at $\epsilon_{\mathrm{D}}=0$ and $\omega=0$. The numbers on the two axes in the bottom planes represent the indices $i$ and $j$ of density matrix element $\rho_{i j}$ or $\rho_{i j}^{(n)}$, respectively.

In the TDHF approach we obtain closed equations of motion for the expectation value $\rho_{n, m}(t)=\left\langle\Psi(t)\left|a_{m}^{+} a_{n}\right| \Psi(t)\right\rangle$, which is the reduced single-electron density matrix, where $\Psi(t)$ is the time dependent Hartree-Fock ground state wave function.

An iterative solution of the TDHF equation provides the density matrix expanded order by order in the external field $E$. The resulting induced charges (diagonal elements of the reduced density matrix) then allow us to calculate the polarizabilities $\alpha, \beta$, and $\gamma$, defined as the expansion coefficients of the induced dipole moment in the electric field. In this work we shall consider the linear polarizability $\alpha(-\omega ; \omega)$, second harmonic polarizability $\beta(-2 \omega ; \omega, \omega)$, and third harmonic polarizability $\gamma(-3 \omega ; \omega, \omega, \omega)$. For brevity we shall also refer to these polarizabilities as $\alpha, \beta$, and $\gamma$, respectively.

In Figure 4 we plot the density matrices $\bar{\rho}, \rho^{(1)}, \rho^{(2)}$, and $\rho^{(3)}$ for $N=38$ at $\epsilon_{\mathrm{D}}=\epsilon_{\mathrm{A}}=0$ where $\bar{\rho}, \rho^{(1)}, \rho^{(2)}$ and $\rho^{(3)}$ are the zeroth-, first-, second-, and third-order expansions of density matrix $\rho$ in $E$, i.e., $\rho=\bar{\rho}+\rho^{(1)}+\rho^{(2)}+\rho^{(3)}+\ldots$ It is particularly instructive to watch the behavior along the "antidiagonal" section reflecting electronic coherence. The HartreeFock ground state $(\bar{\rho})$ is almost diagonal with only the nearest neighbor off-diagonal elements. With increasing order, electronic coherence is gradually built in and the off-diagonal elements of $\rho$ become larger. The high peak in the top panel represents the electron density at the donor.

$\mathrm{BOA}$ is the key parameter determining the bonding structure and the off-resonant polarizabilities. ${ }^{7,13}$ In Figures 5-7, we display $\alpha, \beta$, and $\gamma$ vs BOA for different sizes. For the small size range $N=10 \rightarrow 20, \alpha$ peaks at $\mathrm{BOA} \approx 0, \beta \approx 0$ at $\mathrm{BOA}$ $\approx 0,|\beta|$ peaks at $\mathrm{BOA} \approx \pm 0.2, \gamma$ peaks at $\mathrm{BOA} \approx \pm 0.3$, and $\gamma \approx 0$ at $\mathrm{BOA} \approx \pm 0.2$ and reaches its minimum at $\mathrm{BOA} \approx 0$. This agrees with our previous observation for substituted hexatriene $(N=8) .{ }^{13}$ Similar behaviors for $N=6 \rightarrow 16$ were found in ref 19.

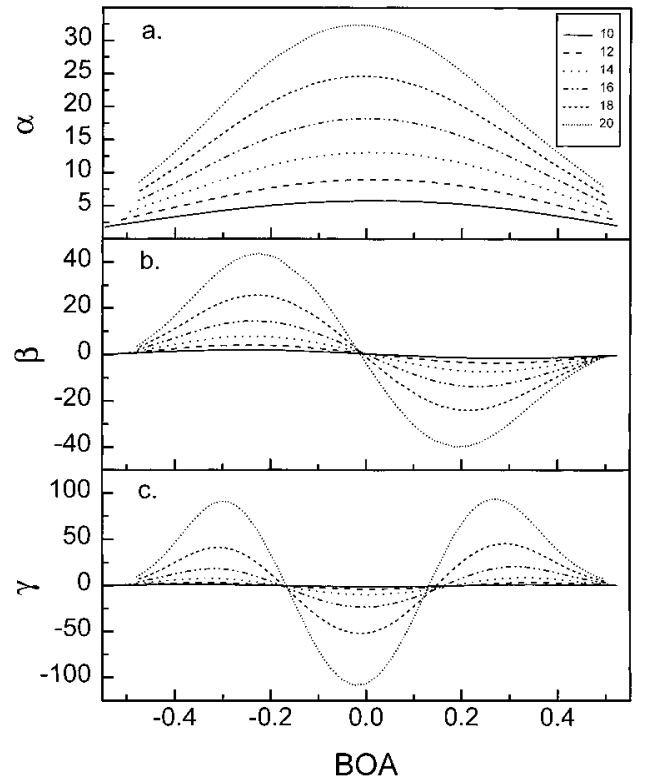

Figure 5. Off-resonant polarizabilities $\alpha, \beta$, and $\gamma$ vs BOA for size $N$ $=10 \rightarrow 20 . \alpha, \beta$, and $\gamma$ are in units of $\mathrm{e} \AA^{2} \mathrm{~V}^{-1}$, e $\AA^{3} \mathrm{~V}^{-2}$, and $\mathrm{e} \AA^{4}$ $\mathrm{V}^{-3}$, respectively. To convert them into esu, they should be multiplied by $1.441 \times 10^{-23}, 4.323 \times 10^{-29}$, and $1.297 \times 10^{-34}$, respectively. All off-resonant polarizabilities are calculated at the frequency $\omega=0.1$ $\mathrm{eV}$, and the damping constant is $0.001 \mathrm{eV}$.

For longer chains $N=22 \rightarrow 38$, the magnitudes of $\alpha, \beta$, and $\gamma$ increase rapidly as BOA is varied in the vicinity of the extreme values of \pm 0.5 . This is similar to the behavior of shorter chains. However, in contrast with the shorter chain, BOA can no longer be varied continuously and an intermediate range of BOA is inaccessible. This reflects the limited ability of the donoracceptor substitution to tune the structure for larger chains. An interesting consequence of this is that for $N \geq 22, \gamma$ is always 


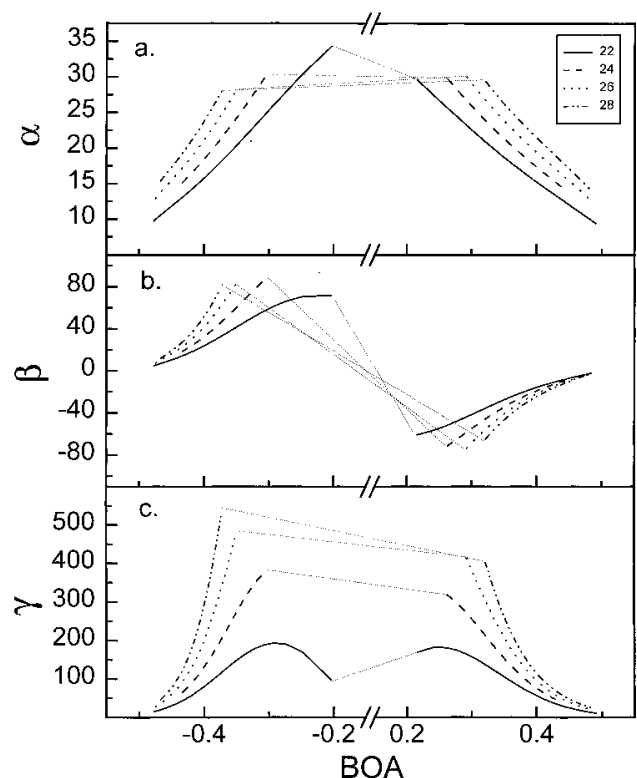

Figure 6. Same as Figure 5 except that $N=22 \rightarrow 28$. The dotted line represents a forbidden range between two branches and is to guide the eyes.

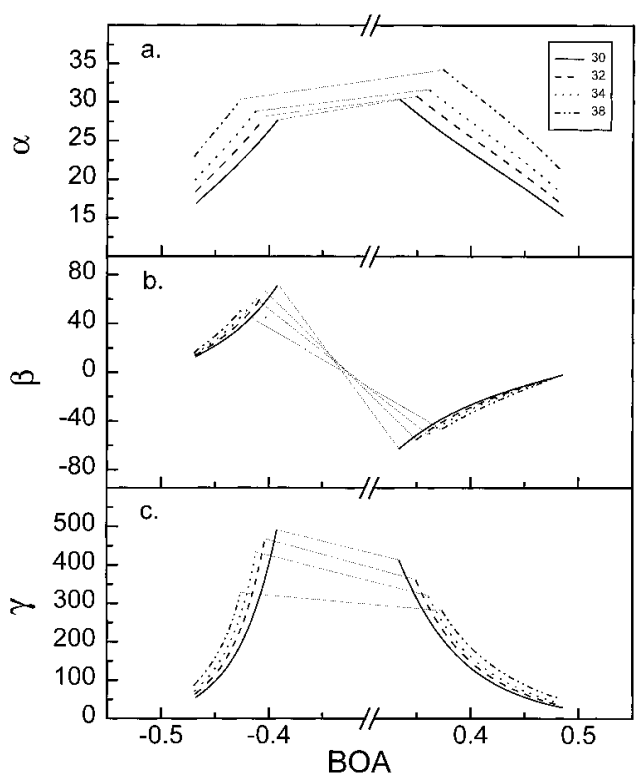

Figure 7. Same as Figure 6 except that $N=30 \rightarrow 38$.

positive. In the vicinity of zero BOA, which is realized for the shorter chains, $\gamma$ is negative.

A discussion of the sign of $\gamma$ and its relation to the electron correlation strength was made by Garito. ${ }^{3}$ Our calculations show that BOA is the fundamental parameter controlling the sign.

In Figures 8-10 we compare the variation of $\alpha, \beta$, and $\gamma$ with BOA for the ground state (solid lines) and the metastable (dashed lines) structures in the size range $N=22$ to 38. Dotted lines indicate the forbidden range of BOA, which cannot be realized by either the ground states or the metastable states. Note that the ground state and the metastable state for a given $\epsilon_{\mathrm{D}}$ have different BOAs so that a given BOA corresponds only to a ground state or to a metastable state. The metastable structures show considerably larger polarizabilities. For $N=$ $30 \rightarrow 38$, the difference can reach $1-2$ orders of magnitude. For instance, for $N=38$, the largest $\gamma$ is about 80000 e $\AA^{4}$ $\mathrm{V}^{-3}$ for the metastable state but only $\sim 300 \mathrm{e}^{4} \mathrm{~V}^{-3}$ for the ground state.
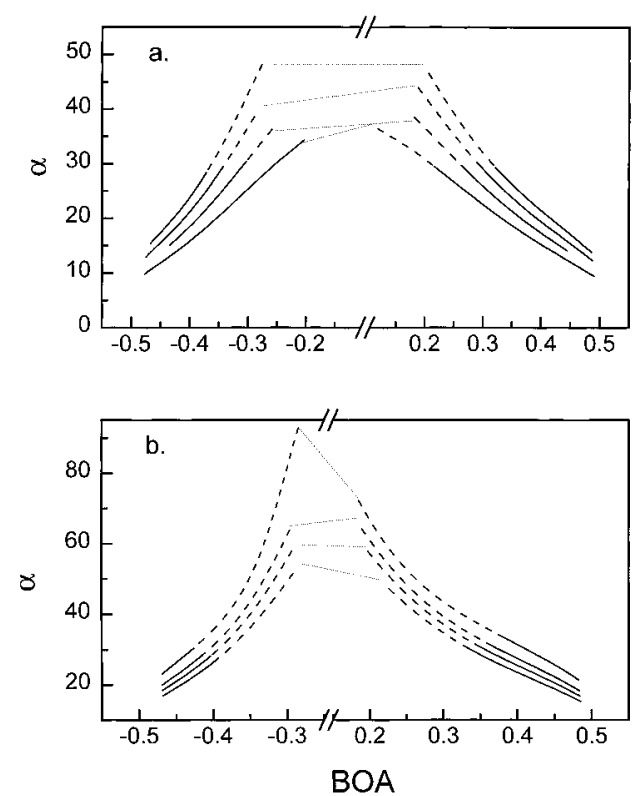

Figure 8. The polarizability $\alpha$ vs BOA for different systems $(N=22$ $\rightarrow 38$ ): (a) $N=22,24,26$, and 28 from bottom to top; (b) $N=30,32$, 34 , and 38 from bottom to top. The solid line is for the ground state. The dashed line is for the metastable state. Parameters and units are the same as in Figure 5.
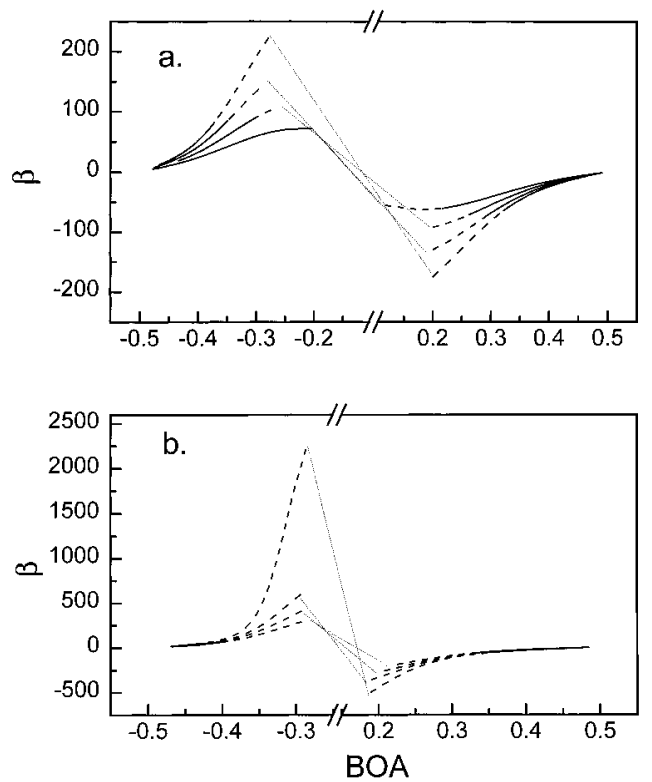

Figure 9. $\beta$ vs BOA for different $N$ : (a) $N=22,24,26$, and 28 from bottom to top (left branch); (b) $N=30,32,34$, and 38 from bottom to top (left branch). Parameters and units are the same as in Figure 8.

\section{Frequency-Dispersed Polarizabilities}

In ref 13 , we compared the off-resonant $\alpha, \beta$, and $\gamma$ of a donor-acceptor substituted hexatriene $(N=8)$ with varying donor-acceptor strengths and those of a donor-acceptor hexatriene subjected to a strong external electric field $E_{\text {ex }}$. Here, we make a similar comparison for the resonant case. In Figures $11-13$, we plot the imaginary parts of resonant polarizabilities $\alpha(-\omega ; \omega), \beta(-2 \omega ; \omega, \omega)$, and $\gamma(-3 \omega ; \omega, \omega, \omega)$ vs $\omega$ for $N=10$ at different values of $\operatorname{BOA}(0.4,0.1,0$, and -0.3$)$. In all calculations of $\alpha(-\omega ; \omega)$ and almost all calculations of $\beta(-$ $2 \omega ; \omega, \omega)$ (except for BOA $=0$ ) the two models yield almost identical answers. In the vicinity of $\mathrm{BOA}=0, \beta \approx 0$ and its precise value is very sensitive to the details of density matrix. It is not surprising that the two $\beta(-2 \omega ; \omega, \omega)$ differ at the resonant frequency $\omega \approx 2.6 \mathrm{eV}$. At $\mathrm{BOA}=-0.3$ the two 

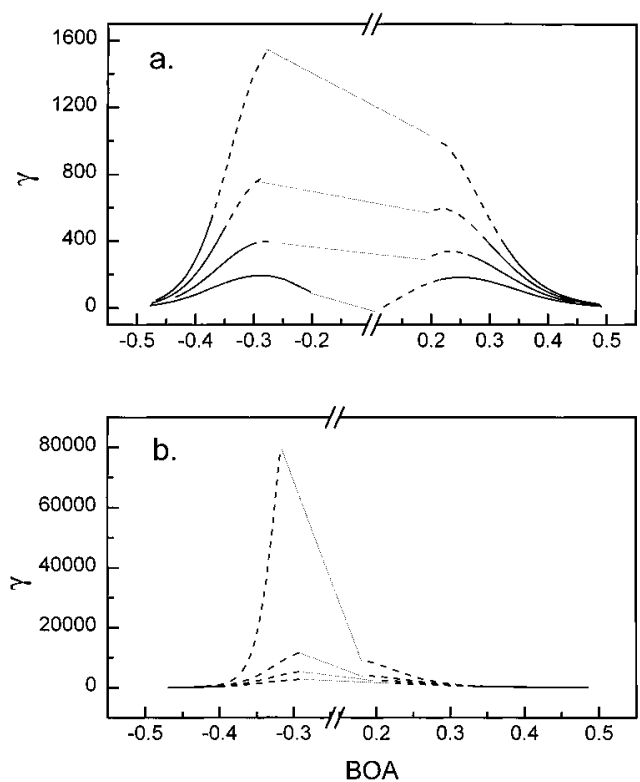

Figure 10. $\gamma$ vs BOA for different $N$ : (a) $N=22,24,26$, and 28 from bottom to top; (b) $N=30,32,34$, and 38 from bottom to top. Parameters and units are the same as in Figure 8.

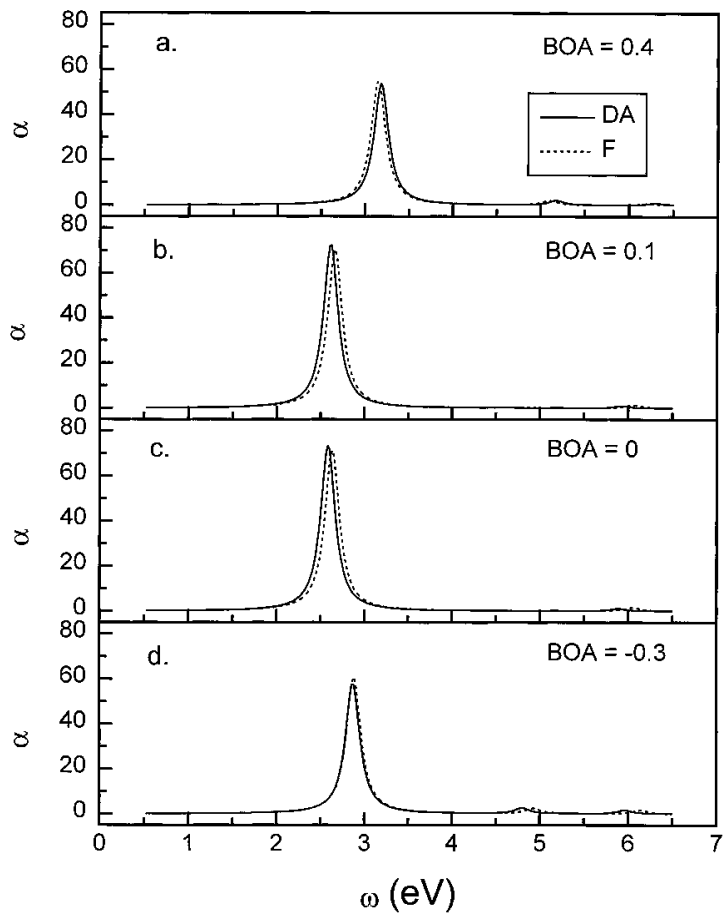

Figure 11. $\operatorname{Im}[\alpha(-\omega ; \omega)]$ vs $\omega$ for $N=10$ (in e $\AA^{2} \mathrm{~V}^{-1}$ ). The solid lines are for different donor-acceptor substitutions. The dashed lines are for the field-driven systems. The damping constant is $0.1 \mathrm{eV}$. The parameters $\left(\epsilon_{D}, E_{e x}\right)$ in parts $\mathrm{a}-\mathrm{d}$ are $(3.7,-0.55),(2.1,-0.31),(1.6$, $-0.24)$, and $(-0.6,0.08)$, respectively. $\epsilon_{\mathrm{D}}$ and $E_{\mathrm{ex}}$ are in units of $\mathrm{eV}$ and V/A, respectively.

curves for $\gamma(-3 \omega ; \omega, \omega, \omega)$ differ near the resonant frequency $\omega$ $\approx 3 \mathrm{eV}$ both in magnitude and in shape. This suggests that higher polarizabilities are more sensitive to details of the system.

\section{Discussion}

Compared with the conventional sum-over-states (SOS) method, the TDHF is much more efficient computationally and enables us to investigate nonlinear optical processes of larger systems. Previously, Marder and co-workers ${ }^{19}$ have investigated the structure-polarizability relationship for conjugated polyenes with up to $N=16$. The present study, which includes systems

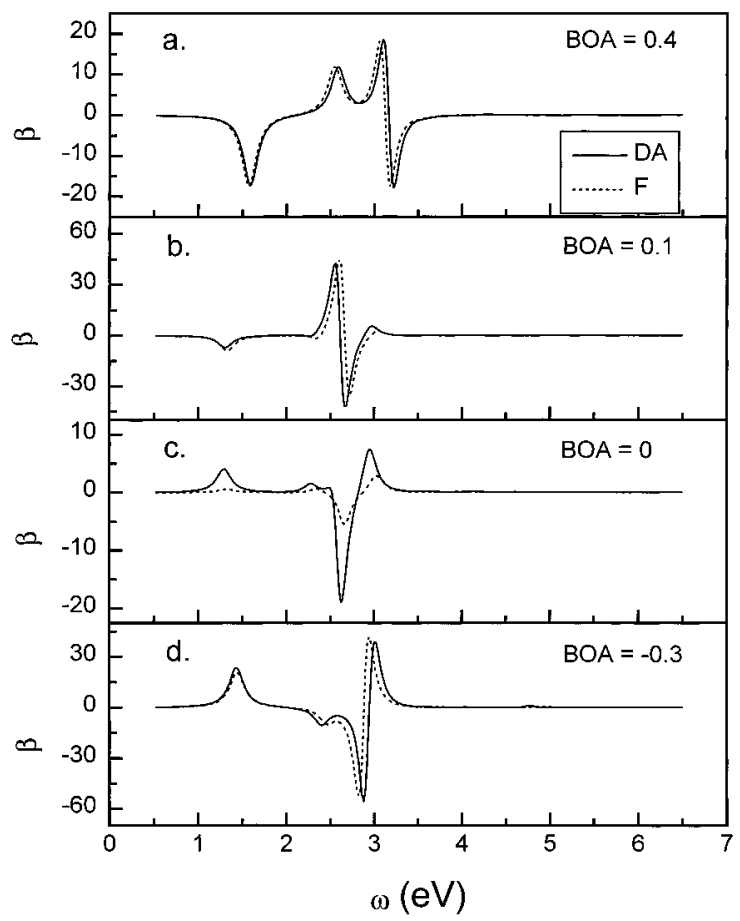

Figure 12. $\operatorname{Im}[\beta(-2 \omega ; \omega, \omega)]$ vs $\omega$ for $N=10$ (in e $\AA^{3} \mathrm{~V}^{-2}$ ). Parameters are the same as in Figure 11.

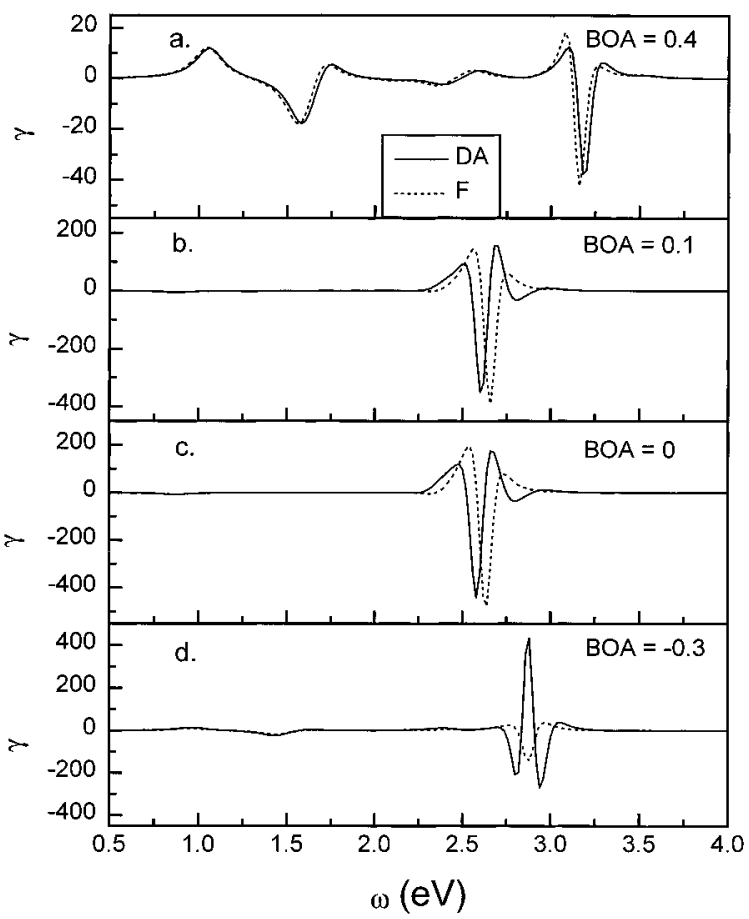

Figure 13. $\operatorname{Im}[\gamma(-3 \omega ; \omega, \omega, \omega)]$ vs $\omega$ for $N=10$ (in e $\AA^{4} \mathrm{~V}^{-3}$ ). Parameters are the same as in Figure 11.

as large as $N=38$, allows us to make several interesting observations.

The most important result is the prediction of metastable structures for larger systems $(N \geq 22)$. Polyacetylene with infinite length has a doubly degenerate ground state with reverse bond alternation. For a finite polyene, the ground and metastable state structures are A-like and C-like (see Figure 1). For $N \leq 20$, only one structure is stable. For $N \geq 22$, for a certain range of $\epsilon_{\mathrm{D}}$ both structures (A and $\mathrm{C}$ ) are stable. These correspond to the two degenerating ground state structures of polyacetylene. One important observation of this work is that this metastable structure has a much larger $\beta$ and $\gamma$ than the 
ground state structure. This is because the metastable states access the conformations with less $|\mathrm{BOA}|$. It would thus be desirable to synthesize these metastable structures. For large systems $N=30 \rightarrow 38$ the magnitudes of $\alpha, \beta$, and $\gamma$ vary rapidly when BOA is tuned near \pm 0.5 . This reflects the important effects of configuration on polarizabilities. The estimated energy barrier is about $0.06 \mathrm{eV}$ or $700 \mathrm{~K}$ for $N=24$. The energy barriers calculated in this study are along the onedimensional path specified in section II. The real energy barriers may be lower in the multidimensional coordinate space. Therefore, the values of energy barriers given in Figure 3 are only upper limits. These observations call for a more careful investigation of structural fluctuation effects. This can be accomplished by e.g., combining the TDHF with Car-Parrinello ab initio molecular dynamics. ${ }^{20}$

BOA is an important factor in determining the signs and magnitudes of the off-resonant polarizabilities., ${ }^{72,13}$ To increase the magnitude of $\beta$, one needs to tune the structure toward BOA $\approx \pm 0.2$, while for $\gamma$, the optimal structures are $\mathrm{BOA} \approx \pm 0.3$ and 0 . As $N$ increases, the influence of a donor-acceptor substitution decreases. For $N \geq 22$, a range of BOA cannot be achieved, which implies that $\gamma$ is always positive. This provides a limitation of donor-acceptor substitution as a means to control the values of hyperpolarizabilities. Gorman and Marder used AM1 and a finite field method and observed that for $N=16$ some range of the bond-length-alternation (BLA) cannot be obtained. ${ }^{19}$ We attribute this small difference to the different Hamiltonians and methods employed in the two studies.

Meyers et al. have investigated the second and third harmonic generation near resonances and asserted that the largest polarizabilities at near-resonance correspond to the same molecular structures displaying the largest off-resonance values. ${ }^{21}$ By comparing the second and third harmonic generation spectra of the two different donor-acceptor substituted systems $(N=$ 10), we demonstrated that BOA is an essential parameter in determining the signs and magnitudes of the resonant polarizabilities as well. In this paper we study only the symmetric substitution $\epsilon_{\mathrm{D}}=-\epsilon_{\mathrm{A}}$. For more general cases, $\alpha, \beta$, and $\gamma$ vs BOA should exhibit similar behaviors, and our main conclusions are expected to hold.
Acknowledgment. We gratefully acknowledge the support of the Air Force Office of Scientific Research and the National Science Foundation.

\section{References and Notes}

(1) (a) Etemad S.; Soos, Z. G. In Spectroscopy of Advanced Materials; Clark, R. J. H., Hester, R. E., Eds.; Wiley: New York, 1991; p 87. (b) Soos, Z. G.; Ramasesha, S.; Galvao, D. S.; Etemad, S. Phys. Rev. B 1993, 47, 1742.

(2) André, J. M.; Delhalle, J.; Brédas, J. L. In Quantum Chemistry Aided Design of Organic Polymers. An Introduction to the Quantum Chemistry of Polymers and its Applications; World Scientific: Singapore, 1991.

(3) Garito, A.; Shi, R. F.; Wu, M. In Phys. Today 1994, 47, 51.

(4) Kohler, B. E.; Spangler, C. W.; Westerfield, C. J. Chem. Phys. 1991, 94, 908

(5) Aramaki, S.; Torruellas, W.; Zanoni, R.; Stegeman, G. I. Opt. Commun. 1991, 82, 94.

(6) Samuel, I. D. W.; Ledoux, I.; Dhenaut, C.; Zyss, J.; Fox, H. H.; Schrock, R. R.; Silbey, R. J. Science 1994, 265, 1070.

(7) Marder, S. R.; Gorman, C. B.; Meyers, F.; Perry, J. W.; Bourhill, G.; Brédas, J. L.; Pierce, B. M. Science 1994 265, 632.

(8) Sekino, H.; Bartlett, R. J. J. Chem. Phys. 1986, 85, 976.

(9) Ring, P.; Schuck, P. The Nuclear Many-Body Problem; Springer: New York, 1980

(10) Rowe, D. J. Rev. Mod. Phys. 1968, 40, 153.

(11) (a) Takahashi, A.; Mukamel, S. J. Chem. Phys. 1994, 100, 2366. (b) Mukamel, S.; Takahashi, A.; Wang, H. X.; Chen, G. Science 1994, $266,251$.

(12) (a) Chen, G.; Mukamel, S. J. Am. Chem. Soc. 1995 117, 4945. (b) Chen, G.; Mukamel, S. Chem. Phys. Lett. 1995 240, 296.

(13) Chen, G.; Mukamel, S. J. Chem. Phys. 1995 103, 9355.

(14) Ohmine, I.; Karplus, M.; Schulten, K. J. Chem. Phys. 1978, 68, 2298.

(15) Fukutome, H. J. Mol. Struct.: THEOCHEM. 1989, 188, 337, and references therein.

(16) Ladik, J. Electronic Structure of Polymers and Molecular Crystals; Plenum Press: New York, 1975.

(17) Chemla, D. S.; Zyss, J. Nonlinear Optical Properties of Organic Molecules and Crystals; Academic: New York, 1987.

(18) Blanchard-Desce, M.; Lehn, J.-M.; Barzoukas, M.; Ledoux, I.; Zyss, J. Chem. Phys. 1994, 181, 281.

(19) Gorman, C. B.; Marder, S. R. Chem. Mater. 1995, 7, 215.

(20) Car, R.; Parrinello, M. Phys. Rev. Lett. 1985, 55, 2471.

(21) Meyers, F.; Marder, S. R.; Pierce, B. M.; Brédas, J. L. J. Am. Chem. Soc. 1994, 116, 10703.

JP9602235 